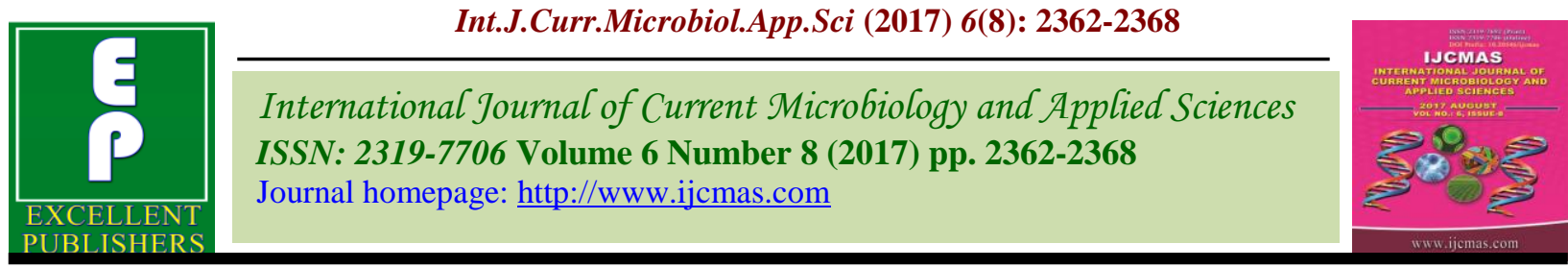

Review Article

https://doi.org/10.20546/ijcmas.2017.608.279

\title{
Promising Epigenetic Approaches Targeting TH17 in Autoimmune Diabetes among Chronic Hepatitis C Infection
}

\author{
Helal F. Hetta ${ }^{*}$ and Azza ElKady ${ }^{2}$ \\ ${ }^{1}$ Department of Medical Microbiology and Immunology, Faculty of Medicine, \\ Assiut University, Assiut, Egypt \\ ${ }^{2}$ Sohag general hospital, Sohag University, Sohag, Egypt \\ *Corresponding author
}

\section{A B S T R A C T}

Keywords

HCV, TH17,

IL17, IL23, T1D,

LADA, mi-RNA,

Epigenetics.

Article Info

Accepted:

21 June 2017

Available Online:

10 August 2017
The revolution of Epigenetics mechanisms including mi-RNA, DNA methylation and histone modification has drawn our attention to target Th17 cell regulatory cytokines and transcription factors which in turn will inhibit the production of interleukin 17 (IL17)- as it's the key proinflammatory cytokine secreted by TH17 cells. The importance of targeting TH17 pathways in autoimmune diabetes come with the deeply ingrained idea of the great role of TH17 in immune pathogenesis of autoimmune diseases. Hepatitis $\mathrm{C}$ virus is one of the most common environmental factors which could trigger autoimmune diabetes by inducing local inflammation with cytokine and chemokine secretion (also called "bystander mechanism".

\section{Introduction}

Diabetes mellitus and hepatitis $\mathrm{C}$ virus infection are two important public health problems associated with high morbidity and mortality (Antonelli et al., 2014).

Globally, Approximately 200 million people become infected with $\mathrm{HCV}$ and 350,000 people per year die from the disease (AbdelHameed et al., 2016; Hetta, 2014; Hetta et al., 2015; Mehta et al., 2016; Shata et al., 2013).

It has been reported that patients with chronic hepatitis $\mathrm{C}$ virus infection are more liable for diabetes mellitus than other liver diseases including hepatitis B virus infection (Hammerstad et al., 2015).
Even though $\mathrm{HCV}$ is a hepatotropic pathogen, the antigen and viral sequences have been detected in pancreases of HCV infected patients which suggested that the infected pancreas might act as a reservoir for $\mathrm{HCV}$ and may play a role in persistence of infection and in turn triggering islet autoimmunity (Hetta et al., 2016; Hieronimus et al., 1997).

Also it is possible that it can promote autoimmunity by inducing local inflammation with cytokine and chemokine secretion (also called "bystander mechanism") (Hammerstad et al., 2015; Helal F. Hetta and Tohamy A. Tohamy, 2017; Yan et al., 2000). 
The American Diabetes association classification, which is based on the etiological criteria has defined three types of diabetes type 1 diabetes mellitus (T1DM), type 2 diabetes mellitus(T2DM), and the third type is Latent autoimmune diabetes of the adult (LADA), sometimes called Type 1.5 diabetes as it is considered as subtype of Type 1 diabetes, is an autoimmune diabetes defined by adult-onset, presence of diabetes associated autoantibodies, and no insulin treatment requirement for a period after diagnosis (Laugesen et al., 2015).In general it is the most prevalent type of autoimmune diabetes.

Among HCV infection Type 2 diabetes may go undiagnosed for autoimmune $\beta$ cell alterations and as misdiagnosed as type 2 diabetes mellitus.

The diagnosis of LADA in HCV infected patients based on their age and time of diagnosis (Antonelli et al., 2014; Gale, 2005).

\section{Lack of understanding LADA or slow- onset type 1 diabetes mellitus}

Phenotypically, LADA is usually misdiagnosed as type 2 diabetes, actually LADA share genetic features with type 1 and type 2 diabetes, Also LADA has worse HbAlc levels than type 2 diabetes.

Immunologically, GAD, glutamic acid decarboxylase autoantibodies are the most common marker in adult-onset diabetes.

Also, islet cells (ICA), insulin-associated antigen (IA2A), or insulin (IAA) and ZnT8 antibodies can be detected in LADA.

Clinically, LADA patients tend to have a lower mean age at diabetes onset, lower body mass index and more frequent need for insulin treatment than patients with type 2 diabetes.
As it is known that Type 1 diabetes mellitus is caused by T-cell mediated destruction of insulin-producing cells. The role of Th17 cells in autoimmune diseases is thought to be associated with their ability to promote inflammatory cytokines and chemokines production by macrophages and other phagocytes which is usually mediated by its key pro-inflammatory cytokine IL17a (Helal F. Hetta, 2017; Ryba-Stanisławowska et al., 2013).Targeting TH17 cells has received intense attention of researchers and clinicians alike with documented effects in inflammation and autoimmune diseases. Herein we will discuss in brief the different new epigenetic mechanism of targeting TH17 pathways which is suggested to be helpful for eradication of autoimmune diabetes among $\mathrm{HCV}$ infected patients.

\section{TH17 and autoimmune diabetes}

Nowadays, the discovery of TH17 cells and its important role in immune pathogenesis of several autoimmune diseases have changed the concept of the implication of TH1 cells in immune pathogenesis of autoimmune diseases(Zambrano-Zaragoza et al., 2014).

TH17 are usually associated with the transcription factor ROR $\gamma \mathrm{t}$ and secretion of the pro-inflammatory cytokines IL-17 (IL17A) and IL-17F (Emamaullee et al., 2009).

Because Th17 subsets are increasingly considered to be a key mediator of all autoimmune disease, all the great efforts now focusing on the new therapeutic strategies designed to inhibit these cells are likely to be applicable in type 1 diabetes or type 1.5 diabetes.

Since 2003, the discovery of IL23 and its great role in promoting autoimmune diseases as it serves as a pivotal factor that initiate differentiation and inflammatory function of 
pathogenic TH17 as it is critical for expansion and survival of TH17 cells. Interaction of IL23-producing APCs and Th17 cells has been shown to have a role in many autoimmune diseases. In support of this view, all the immunologist trials is targeting IL-23 pathway, IL-17 production or action by using IL-17R antagonist and IL-17A-blocking antibodies have been shown to attenuate autoimmune diseases(Gaffen et al., 2014). A recent study has reported that the combination of inhibition of IL-23 and IL-17 is more potent in treating Th17-mediated autoimmunity in mouse models (Mangan et al., 2015).

\section{Epigenetic regulation of TH17 cells}

There are three main epigenetic modifications, including DNA methylation, histone modification, and microRNA. All of them are associated with transcriptional regulation and determination of the cellular transcriptome, thereby contributing to cell function (Lee et al., 2009) as shown in figure 1 .

Fig.1 Different epigenetic mechanisms that regulate TH17 differentiation. MicroRNA 21 is highly upregulated in Th17 cells and increase expression of IL17. MicroRNA 16 control the balance between iTreg and TH17 by affecting the expression of ROR $\gamma \mathrm{t}$ and FoxP3.MicrRNA 155 enhance TH17 cells differentiation and increase IL17 production. MicroRNA 30a regulates Th17 differentiation by inhibiting the translation of IRF-4 factor which in turn facilitate ROR- $\gamma$ recruitment and binding to TH17 cells. Histone modification control TH17 differentiation by increase exhibition high levels of $\mathrm{H} 3 \mathrm{~K} 27 \mathrm{Ac}$ and $\mathrm{H} 3 \mathrm{~K} 4 \mathrm{me} 3$ which are two active regulations during Th17 cell differentiation. TRABID which is one of deubiquitinating enzymes impaired the induction of IL23 which in turn impair production of TH17. DNA methylation is also important for the generation and plasticity of Th17 and iTreg cells

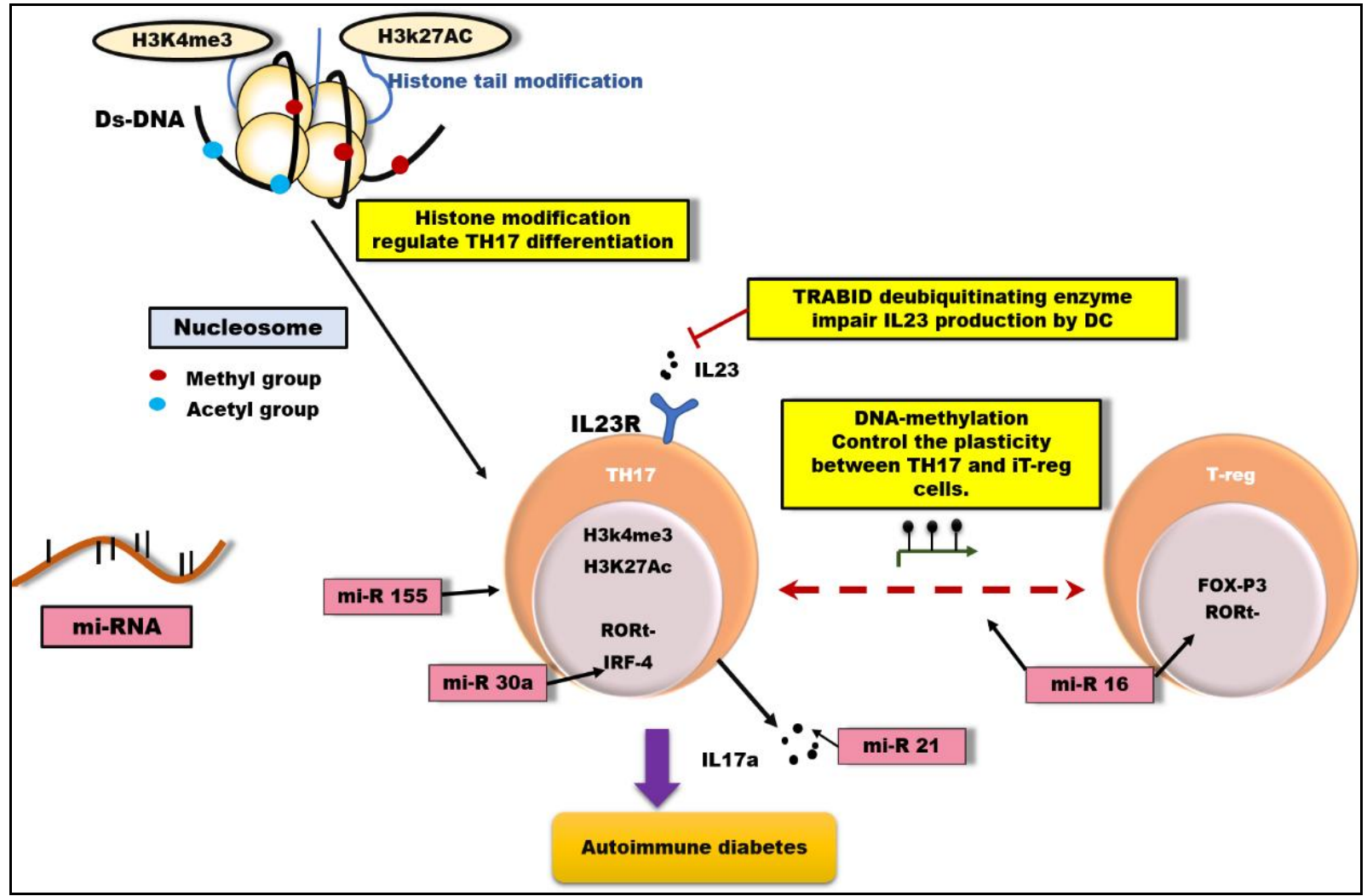




\section{mi-RNA and TH17 cells}

There is no doubt that miRNA is able to regulate the expression of several (maybe even hundreds of) target genes and are involved in important processes, such as embryonic development, immune response, inflammation, and oncogenesis, as well as cellular growth and proliferation. Positive correlations in miRNA expression and IL-17 levels have been observed in different studies(Khan \& Ansar Ahmed, 2015).

Emerging studies indicate that miR-21 promotes inflammation and plays important roles during the pathogenesis of autoimmune disease including type 1 diabetes (T1D) (Osipova et al., 2014). In an interesting study, Murugaiyan et al., found that miR-21 expression is elevated in Th17 cells and that miR-21-deficient mice produce fewer Th17 cells and are resistant to EAE(Murugaiyan et al., 2015). Another recent study, Dong et al., have reported that miR-21 negative regulate the TH17 differentiation in PBMCs of RA patients.(Dong et al., 2014).Also Wu YH et al., have found that MI-R16 may be involved in Th17/Treg imbalance of RA patients by affecting the expression of ROR $\gamma \mathrm{t}$ and FoxP3 (Wu et al., 2016).

Ryan O'Connell et al., have shown that miR155 plays an important role in driving chronic inflammation that is inappropriately directed at tissue-specific antigens, a destructive process that is at the heart of human autoimmune diseases as miR-155's has positive role in Th17 cell development by blocking the inhibitory impact of cytokines such as IL-4 and IFN- $\gamma$ on the Th17 cell differentiation pathway(O'Connell et al., 2010).

IRF-4, member of interferon regulatory factor family, is one of the pioneer factors that could facilitate $\mathrm{ROR}-\gamma$ recruitment and binding to
TH17 cells. Due to its great role in regulation of the differentiation of TH17 cells, Ming Zhoao et al., put all his focus in his recent study on, MS, multiple sclerosis patients to find the miRNA that could control IRF-4 regulation by using in-depth RNA-sequencing analysis. Finally, he succeed to be the first one which reported that miR-30a regulates Th17 differentiation by inhibiting the translation of IRF-4 factor (Zhao et al., 2016)

\section{Role DNA methylation and histone modification in regulation of TH17cells}

DNA methylation and Histone modifications, which include methylation, acetylation, ubiquitination, phosphorylation, and simulation, are classified as transcriptionally active or repressive markers and one of the epigenetic maintainers (Araki \& Mimura, 2017)

It has become interestingly enough that histone modifications, such as acetylation or methylation, are widely found at many Th17 cell-specific gene loci, including RORC, IL$23 r$, therefore playing significant roles in maintaining Th17 cell chromatin state. The ROR $\gamma \mathrm{t}$ loci is indispensable for Th17 development and exhibits high levels of $\mathrm{H} 3 \mathrm{~K} 27 \mathrm{Ac}$ and $\mathrm{H} 3 \mathrm{~K} 4 \mathrm{me} 3$, which are two active regulations during Th17 cell differentiation, as well proved to be marked at the IL17a and IL17f loci (Wang et al., 2016), (Akimzhanov et al., 2007).

Also in an interesting study highlighted an epigenetic mechanism for regulation IL12 and IL23. The deficiency of Trabid which is TRAF-binding domain (TRABID) and one of deubiquitination enzymes-in DCs and macrophages impaired the induction of $\mathrm{Il12}$ and Il23 which in turn impair production of the TH1 and TH17 subsets of inflammatory T cells and this could render autoimmune encephalomyelitis as Trabid regulates histone 
modifications at the I112 and IL23 promoter by controlling the fate of the histone demethylase Jmjd2d(Jin et al., 2016).

DNA methylation is also important for the generation and plasticity of Th17 and iTreg cells. The IL17a gene remains hypermethylated in naive CD4+ T cells and is only demethylated in IL17A-producing effector cells. The recent evidence indicates that the chromatin structure of the I117a, II17f, IFNG, and ROR $\gamma$ t loci in Th17 cells alter rapidly in response to cell-extrinsic factors, thereby showing a particularly high degree of plasticity.

Th17 cells have the capacity to express IFN-g and Il17. The differentiation of Th17 into stabilized Treg cells was usually associated with stable FoxP3 demethylation and obtain suppressive function. Interestingly, Th17derived regulatory $\mathrm{T}$ cells (Tregs) were resistant to Th17 reconversion, which could have important clinical implications for immunotherapy.(Singh et al., 2014; SuarezAlvarez et al., 2012)

In conclusion, the recent discoveries in the field of epigenetic including the three main mechanisms mi-RNA, DNA methylation and histone modification have improved our knowledge in immunotherapy for diabetes mellitus type 1 and type 1.5 which is considered one the most common extrahepatic endocrine manifestation. Due to its pivotal role in regulation of TH17 cells which play the major role in the pathogenesis of diabetes mellitus type 1 .

Signature miRNA profile can be potentially used as novel biomarkers for Th17-mediated immune reactions. However, more in-depth and mechanistic studies are required to further define the role of miRNAs in IL-17 induction and interplay of miRNA with IL-17-related transcription factors and signaling pathways

\section{Conflict of Interest}

The authors declare that they do not have conflict of interests.

\section{Acknowledgement}

We thank the Grant office, Faculty of Medicine, Assiut University, Egypt for their financial support.

\section{References}

Abdel-Hameed, E. A., Rouster, S. D., Ji, H., Ulm, A., Hetta, H. F., Anwar, N., Sherman, K. E. \& Shata, M. T. (2016). Evaluating the Role of Cellular Immune Responses in the Emergence of HCV NS3 Resistance Mutations During Protease Inhibitor Therapy. Viral immunology29, 252-258.

Akimzhanov, A. M., Yang, X. O. \& Dong, C. (2007). Chromatin remodeling of interleukin-17 (IL-17)-IL-17F cytokine gene locus during inflammatory helper $\mathrm{T}$ cell differentiation. The Journal of biological chemistry282, 5969-5972.

Antonelli, A., Ferrari, S. M., Giuggioli, D., Di Domenicantonio, A., Ruffilli, I., Corrado, A., Fabiani, S., Marchi, S., Ferri, C. \& other authors (2014). Hepatitis C virus infection and type 1 and type 2 diabetes mellitus. World Journal of Diabetes5, 586-600.

Araki, Y. \& Mimura, T. (2017). The Histone Modification Code in the Pathogenesis of Autoimmune Diseases. Mediators of Inflammation 2017, 12.

Dong, L., Wang, X., Tan, J., Li, H., Qian, W., Chen, J., Chen, Q., Wang, J., Xu, W. \& other authors (2014). Decreased expression of microRNA-21 correlates with the imbalance of Th17 and Treg cells in patients with rheumatoid arthritis. Journal of cellular and molecular medicine 18, 2213-2224.

Emamaullee, J. A., Davis, J., Merani, S., Toso, C., Elliott, J. F., Thiesen, A. \& Shapiro, 
A. M. J. (2009). Inhibition of Th17 Cells Regulates Autoimmune Diabetes in NOD Mice. Diabetes58, 1302-1311.

Gaffen, S. L., Jain, R., Garg, A. V. \& Cua, D. J. (2014). The IL-23-IL-17 immune axis: from mechanisms to therapeutic testing. Nat Rev Immunol14, 585-600.

Gale, E. A. (2005). Latent autoimmune diabetes in adults: a guide for the perplexed. Diabetologia48, 2195-2199.

Hammerstad, S. S., Grock, S. F., Lee, H. J., Hasham, A., Sundaram, N. \& Tomer, Y. (2015). Diabetes and Hepatitis C: A TwoWay Association. Frontiers in Endocrinology6.

Helal F. Hetta, A. E., Khairy H. Morsy, Ismael S. Mohamed (2017). Serum Level of IL17a among Cirrhotic Hepatitis C Virus Infected Patients with Incidence of Diabetes Mellitus. THE EGYPTIAN JOURNAL OF IMMUNOLOGY24.

Helal F. Hetta, A. E., Khairy H. Morsy,Ismael S Mohamed, Khaled M. Hassanein, \& Tohamy A. Tohamy, H. M. E. a. G. A. M. (2017). Circulating IL17a and IFNGamma Serum Levels in Cirrhotic Hepatitis C Virus Infected Patients with Autoimmune

Thyroiditis. IntJCurrMicrobiolAppSci6, 19721983.

Hetta, H. F. (2014). Gut immune response in the presence of hepatitis $\mathrm{C}$ virus infection. World Journal of Immunology4, 52.

Hetta, H. F., Mekky, M. A., Khalil, N. K., Mohamed, W. A., El-Feky, M. A., Ahmed, S. H., Daef, E. A., Nassar, M. I., Medhat, A. \& other authors (2015). Association of colonic regulatory $\mathrm{T}$ cells with hepatitis $\mathrm{C}$ virus pathogenesis and liver pathology. Journal of gastroenterology and hepatology30, 1543-1551.

Hetta, H. F., Mekky, M. A., Khalil, N. K., Mohamed, W. A., El-Feky, M. A., Ahmed, S. H., Daef, E. A., Medhat, A., Nassar, M. I. \& other authors (2016). Extra-hepatic infection of hepatitis C virus in the colon tissue and its relationship with hepatitis $\mathrm{C}$ virus pathogenesis. Journal of medical microbiology65, 703-712.

Hieronimus, S., Fredenrich, A., Tran, A., Benzaken, S. \& Fenichel, P. (1997). Antibodies to GAD in chronic hepatitis C patients. Diabetes Care20, 1044.

Jin, J., Xie, X., Xiao, Y., Hu, H., Zou, Q., Cheng, X. \& Sun, S.-C. (2016). Epigenetic regulation of the expression of Il12 and Il23 and autoimmune inflammation by the deubiquitinase Trabid. Nature immunology.

Khan, D. \& Ansar Ahmed, S. (2015). Regulation of IL-17 in autoimmune diseases by transcriptional factors and microRNAs. Frontiers in Genetics6.

Laugesen, E., Østergaard, J. A. \& Leslie, R. D. G. (2015). Latent autoimmune diabetes of the adult: current knowledge and uncertainty. Diabet Med32, 843-852.

Lee, C. G., Sahoo, A. \& Im, S. H. (2009). Epigenetic Regulation of Cytokine Gene Expression in $\mathrm{T}$ Lymphocytes. Yonsei Medical Journal50, 322-330.

Mangan, P. R., Su, L. J., Jenny, V., Tatum, A. L., Picarillo, C., Skala, S., Ditto, N., Lin, Z., Yang, X. \& other authors (2015). Dual Inhibition of Interleukin-23 and Interleukin-17 Offers Superior Efficacy in Mouse Models of Autoimmunity. $J$ Pharmacol Exp Ther354, 152-165.

Mehta, M., Hetta, H. F., Abdel-Hameed, E. A., Rouster, S. D., Hossain, M., Mekky, M. A., Khalil, N. K., Mohamed, W. A., ElFeky, M. A. \& other authors (2016). Association between IL28B rs12979860 single nucleotide polymorphism and the frequency of colonic Treg in chronically HCV-infected patients. Archives of virology161, 3161-3169.

Murugaiyan, G., da Cunha, A. P., Ajay, A. K., Joller, N., Garo, L. P., Kumaradevan, S., Yosef, N., Vaidya, V. S. \& Weiner, H. L. (2015). MicroRNA-21 promotes Th17 differentiation and mediates experimental autoimmune encephalomyelitis. The Journal of clinical investigation 125 , 1069-1080. 
O'Connell, R. M., Kahn, D., Gibson, W. S. J., Round, J. L., Scholz, R. L., Chaudhuri, A. A., Kahn, M. E., Rao, D. S. \& Baltimore, D. (2010). MicroRNA-155 Promotes Autoimmune Inflammation by Enhancing Inflammatory $\mathrm{T}$ Cell Development. Immunity33, 607-619.

Osipova, J., Fischer, D. C., Dangwal, S., Volkmann, I., Widera, C., Schwarz, K., Lorenzen, J. M., Schreiver, C., Jacoby, U. $\&$ other authors (2014). Diabetesassociated microRNAs in pediatric patients with type 1 diabetes mellitus: a cross-sectional cohort study. The Journal of clinical endocrinology and metabolism99, E1661-1665.

Ryba-Stanisławowska, M., Skrzypkowska, M., Myśliwiec, M. \& Myśliwska, J. (2013). Loss of the balance between CD4+ Foxp3+ regulatory $\mathrm{T}$ cells and $\mathrm{CD} 4+$ IL17A+ Th17 cells in patients with type 1 diabetes. Human Immunology74, 701707.

Shata, M. T., Abdel-Hameed, E. A., Hetta, H. F. \& Sherman, K. E. (2013). Immune activation in $\mathrm{HIV} / \mathrm{HCV}$-infected patients is associated with low-level expression of liver expressed antimicrobial peptide-2 (LEAP-2). Journal of clinical pathology66, 967-975.

Singh, A., Yamamoto, M., Ruan, J., Choi, J. Y., Gauvreau, G. M., O'Byrne, P. M., Olek, S., Hoffmueller, U., Carlsten, C. \& other authors (2014). Th17/Treg ratio derived using DNA methylation analysis discriminates allergen-induced early from dual asthmatic responses. Allergy, Asthma, and Clinical Immunology : Official Journal of the Canadian Society of Allergy and Clinical Immunology10, A46-A46.

Suarez-Alvarez, B., Rodriguez, R. M., Fraga, M. F. \& Lopez-Larrea, C. (2012). DNA methylation: a promising landscape for immune system-related diseases. Trends in genetics : TIG28, 506-514.

Wang, Z., Yin, H., Lau, C. S. \& Lu, Q. (2016). Histone Posttranslational Modifications of CD4(+) $\mathrm{T}$ Cell in Autoimmune Diseases. International Journal of Molecular Sciences 17.

Wu, Y. H., Liu, W., Xue, B., Zhang, L., Liu, X. Y., Liu, B., Wang, Y., Cai, Y. \& Duan, R. (2016). Upregulated Expression of microRNA-16 Correlates with Th17/Treg Cell Imbalance in Patients with Rheumatoid Arthritis. DNA and cell biology35, 853-860.

Yan, F. M., Chen, A. S., Hao, F., Zhao, X. P., Gu, C. H., Zhao, L. B., Yang, D. L. \& Hao, L. J. (2000). Hepatitis C virus may infect extrahepatic tissues in patients with hepatitis C. World journal of gastroenterology6, 805-811.

Zambrano-Zaragoza, J., \#xe9, Francisco, Romo-Mart, \#xed, nez, E. J., Dur, \#xe1, n-Avelar, M. d. J. \& other authors (2014). Th17 Cells in Autoimmune and Infectious Diseases. International Journal of Inflammation2014, 12.

Zhao, M., Sun, D., Guan, Y., Wang, Z., Sang, D., Liu, M., Pu, Y., Fang, X., Wang, D. \& other authors (2016). Disulfiram and Diphenhydramine Hydrochloride Upregulate miR-30a to Suppress IL-17Associated Autoimmune Inflammation. The Journal of Neuroscience36, 9253 9266.

\section{How to cite this article:}

Helal F. Hetta and Azza ElKady. 2017. Promising Epigenetic Approaches Targeting TH17 in Autoimmune Diabetes among Chronic Hepatitis C Infection. Int.J.Curr.Microbiol.App.Sci. 6(8): 2362-2368. doi: https://doi.org/10.20546/ijcmas.2017.608.279 